Many mathematical models give rise to problems for which only nonnegative solutions make sense; therefore, many research articles on the theory of positive solutions have appeared. For a complete overview on this subject, we refer to the monograph [3].

In this paper, we also present, as a consequence of our main theorems, some results on the existence of nonnegative solutions for a particular problem of type

$$
\left\{\begin{array}{l}
\left.-u^{\prime \prime}=\lambda \alpha(x) g(u) \quad \text { on }\right] a, b[ \\
u(a)=u^{\prime}(b)=0,
\end{array}\right.
$$

where $\alpha \in L^{1}([a, b])$ is such that $\alpha(x) \geq 0$ a.e. $x \in[a, b], \alpha \neq \equiv$, and $g: \mathbb{R} \rightarrow \mathbb{R}$ is a nonnegative continuous function. In particular, we obtain for such a problem the existence of at least three nonnegative solutions by requiring that the function $g$ has a superlinear behavior at zero, a sublinear behavior at infinity, and a particular growth in a suitable interval $[c, d]$. By a similar approach, in [4], the authors obtain the existence of multiple solutions for a Neumann elliptic problem.

Multiplicity results for a mixed boundary value problem have been studied by several authors (see, for instance, [5-8] and references therein). In [5], the authors establish multiplicity results for problem (P), when $p=2$, and, in particular, they obtain the existence of three solutions, one of which can be trivial. On the contrary, our results (Theorems 3.5 and 3.6) guarantee the existence of three nonnegative and nontrivial solutions.

In [7], by using a fixed point theorem, the existence of at least three solutions for a mixed boundary problem with the equation $\left(\left|u^{\prime}\right|^{p-2} u^{\prime}\right)^{\prime}=q(x) f(u)$ is obtained, by requiring, among other things, the boundness of $f$ in a right neighborhood of zero (hypothesis (H6), Theorem 3.1), instead in our results (Theorems 3.5 and 3.6) the nonlinearity can blow up at zero.

Here, as an example, we present the following result which is a particular case of Theorem 3.6.

Theorem 1.1 Let $g: \mathbb{R} \rightarrow \mathbb{R}$ be a nonnegative continuous function such that

$$
\lim _{\xi \rightarrow 0^{+}} \frac{g(\xi)}{\xi}=+\infty, \quad \lim _{\xi \rightarrow+\infty} \frac{g(\xi)}{\xi}=0
$$

and

$$
\int_{0}^{1} g(\xi) d \xi<\frac{1}{16} \int_{0}^{2} g(\xi) d \xi
$$

Then, for each $\lambda \in] \frac{8}{\int_{0}^{2} g(\xi) d \xi}, \frac{1}{2 \int_{0}^{1} g(\xi) d \xi}[$, the problem

$$
\left\{\begin{array}{l}
\left.-u^{\prime \prime}=\lambda g(u) \quad \text { in }\right] 0,1[ \\
u(0)=u^{\prime}(1)=0
\end{array}\right.
$$

admits at least three classical nonnegative and nontrivial solutions. 


\section{Preliminaries and basic notations}

Our main tools are Theorems 2.1 and 2.2, consequences of the existence result of a local minimum [9, Theorem 3.1] which is inspired by the Ricceri variational principle (see [10]). For more information on this topic see, for instance, [11] and [12].

Given a set $X$ and two functionals $\Phi, \Psi: X \rightarrow \mathbb{R}$, put

$$
\begin{aligned}
& \beta\left(r_{1}, r_{2}\right)=\inf _{v \in \Phi^{-1}(] r_{1}, r_{2}[)} \frac{\sup _{u \in \Phi^{-1}(] r_{1}, r_{2}[)} \Psi(u)-\Psi(v)}{r_{2}-\Phi(v)}, \\
& \rho_{2}\left(r_{1}, r_{2}\right)=\sup _{v \in \Phi^{-1}(] r_{1}, r_{2}[)} \frac{\Psi(v)-\sup _{\left.\left.u \in \Phi^{-1}(]-\infty, r_{1}\right]\right)} \Psi(u)}{\Phi(v)-r_{1}}
\end{aligned}
$$

for all $r_{1}, r_{2} \in \mathbb{R}$, with $r_{1}<r_{2}$, and

$$
\rho(r)=\sup _{v \in \Phi^{-1}(] r,+\infty[)} \frac{\Psi(v)-\sup _{\left.\left.u \in \Phi^{-1}(]-\infty, r\right]\right)} \Psi(u)}{\Phi(v)-r},
$$

for all $r \in \mathbb{R}$.

Theorem 2.1 [9, Theorem 5.1] Let $X$ be a reflexive real Banach space; $\Phi: X \rightarrow \mathbb{R}$ be a sequentially weakly lower semicontinuous, coercive, and continuously Gâteaux differentiable function whose Gâteaux derivative admits a continuous inverse on $X^{*} ; \Psi: X \rightarrow \mathbb{R}$ be a continuously Gâteaux differentiable function whose Gâteaux derivative is compact. Put $I_{\lambda}=\Phi-\lambda \Psi$ and assume that there are $r_{1}, r_{2} \in \mathbb{R}$, with $r_{1}<r_{2}$, such that

$$
\beta\left(r_{1}, r_{2}\right)<\rho_{2}\left(r_{1}, r_{2}\right)
$$

where $\beta$ and $\rho_{2}$ are given by (2.1) and (2.2).

Then, for each $\lambda \in] \frac{1}{\rho_{2}\left(r_{1}, r_{2}\right)}, \frac{1}{\beta\left(r_{1}, r_{2}\right)}\left[\right.$, there is $u_{0, \lambda} \in \Phi^{-1}(] r_{1}, r_{2}[)$ such that $I_{\lambda}\left(u_{0, \lambda}\right) \leq I_{\lambda}(u)$ for all $u \in \Phi^{-1}(] r_{1}, r_{2}[)$ and $I_{\lambda}^{\prime}\left(u_{0, \lambda}\right)=0$.

Theorem 2.2 [9, Theorem 5.3] Let $X$ be a real Banach space; $\Phi: X \rightarrow \mathbb{R}$ be a continuously Gâteaux differentiable function whose Gâteaux derivative admits a continuous inverse on $X^{*} ; \Psi: X \rightarrow \mathbb{R}$ be a continuously Gâteaux differentiable function whose Gâteaux derivative is compact. Fix $\inf _{X} \Phi<r<\sup _{X} \Phi$ and assume that

$$
\rho(r)>0
$$

where $\rho$ is given by (2.3), and for each $\lambda>\frac{1}{\rho(r)}$, the function $I_{\lambda}=\Phi-\lambda \Psi$ is coercive.

Then, for each $\lambda>\frac{1}{\rho(r)}$, there is $u_{0, \lambda} \in \Phi^{-1}(] r,+\infty[)$ such that $I_{\lambda}\left(u_{0, \lambda}\right) \leq I_{\lambda}(u)$ for all $u \in$ $\Phi^{-1}(] r,+\infty[)$ and $I_{\lambda}^{\prime}\left(u_{0, \lambda}\right)=0$.

Now, consider problem $(\mathrm{P})$ and assume that $q, r \in L^{\infty}([a, b])$ with

$$
\underset{[a, b]}{\operatorname{essinf}} q>0 \text { and } \underset{[a, b]}{\operatorname{essinf}} r \geq 0 \text {. }
$$

Denote by $X=\left\{u \in W^{1, p}([a, b]): u(a)=0\right\}$ endowed with the norm

$$
\|u\|=\left(\int_{a}^{b} q(x)\left|u^{\prime}(x)\right|^{p} d x+\int_{a}^{b} r(x)|u(x)|^{p} d x\right)^{\frac{1}{p}}
$$


Throughout the sequel, $f:[a, b] \times \mathbb{R} \rightarrow \mathbb{R}$ is an $L^{1}$-Carathéodory function. We recall that a function $f:[a, b] \times \mathbb{R} \rightarrow \mathbb{R}$ is said to be an $L^{1}$-Carathéodory function if $x \rightarrow f(x, t)$ is measurable for all $t \in \mathbb{R}, t \rightarrow f(x, t)$ is continuous for almost every $x \in[a, b]$, and for all $M>0$, one has $\sup _{|t| \leq M}|f(x, t)| \in L^{1}([a, b])$. Clearly, if $f$ is continuous in $[a, b] \times \mathbb{R}$, then it is $L^{1}$-Carathéodory.

Put

$$
F(x, \xi):=\int_{0}^{\xi} f(x, t) d t \quad \text { for all }(x, \xi) \in[a, b] \times \mathbb{R} .
$$

Moreover, it is well known that $(X,\|\cdot\|)$ is compactly embedded in $\left(C^{0}([a, b]),\|\cdot\|_{\infty}\right)$ and one has

$$
\|u\|_{\infty}<\left(\frac{b-a}{q_{0}}\right)^{\frac{p-1}{p}}\|u\| \quad \text { for all } u \in X .
$$

We use the following notations:

$$
\|q\|_{\infty}:=\underset{x \in[a, b]}{\operatorname{ess} \sup } q(x), \quad\|r\|_{\infty}:=\underset{x \in[a, b]}{\operatorname{ess} \sup } r(x) .
$$

In order to study problem (P), we introduce the functionals $\Phi, \Psi: X \rightarrow \mathbb{R}$ defined as follows:

$$
\Phi(u)=\frac{1}{p}\|u\|^{p} \quad \text { and } \quad \Psi(u)=\int_{a}^{b} F(x, u(x)) d x, \quad \forall u \in X .
$$

Clearly, the critical points of the functional $\Phi-\lambda \Psi$ on $X$ are weak solutions of problem (P). We recall that $u:[a, b] \rightarrow \mathbb{R}$ is a weak solution of problem (P) if $u \in X$ satisfies the following condition:

$$
\begin{aligned}
& \int_{a}^{b} q(x)\left|u^{\prime}(x)\right|^{p-2} u^{\prime}(x) v^{\prime}(x) d x+\int_{a}^{b} r(x)|u(x)|^{p-2} u(x) v(x) d x \\
& \quad=\lambda \int_{a}^{b} f(x, u(x)) v(x) d x, \quad \text { for all } v \in X .
\end{aligned}
$$

Clearly, if $f$ is continuous, $q \in C^{1}([a, b])$, and $r \in C^{0}([a, b])$, the weak solutions for (P) are classical solutions.

\section{Main results}

In this section we present our main results.

Given two nonnegative constants $c, d$ such that $\bar{q} c^{p} \neq \bar{r} d^{p}$, where

$$
\bar{q}=\frac{1}{p}\left(\frac{q_{0}}{b-a}\right)^{p-1} \text { and } \quad \bar{r}=\frac{1}{p}\left(\frac{2}{b-a}\right)^{p-1}\left[\|q\|_{\infty}+\frac{p+2}{p+1}\left(\frac{b-a}{2}\right)^{p}\|r\|_{\infty}\right] \text {, }
$$

put

$$
a_{d}(c):=\frac{\int_{a}^{b} \max _{|\xi| \leq c} F(x, \xi) d x-\int_{\frac{a+b}{2}}^{b} F(x, d) d x}{\bar{q} c^{p}-\bar{r} d^{p}} .
$$


Theorem 3.1 Under the following conditions:

(i) there exist three constants $c_{1}, c_{2}, d$, with

$$
\left(\frac{q_{0}^{p-2}}{2^{p-1}}\right)^{\frac{1}{p}} c_{1}<d<\left(\frac{q_{0}^{p-1}}{2^{p-1}}\right)^{\frac{1}{p}} \frac{1}{\left[\|q\|_{\infty}+\frac{p+2}{p+1}\left(\frac{b-a}{2}\right) p\|r\|_{\infty}\right]^{\frac{1}{p}}} c_{2},
$$

such that

$$
a_{d}\left(c_{2}\right)<a_{d}\left(c_{1}\right)
$$

and $\int_{a}^{\frac{a+b}{2}} F(x, t) d x \geq 0 \forall t \in[0, d]$;

(ii) there exist $v>p$ and $R>0$ such that

$$
0<v F(x, t) \leq t f(x, t), \quad \text { for all }|t| \geq R \text { and for all } x \in[a, b]
$$

For each $\lambda \in] \frac{1}{a_{d}\left(c_{1}\right)}, \frac{1}{a_{d}\left(c_{2}\right)}\left[\right.$, problem $(\mathrm{P})$ admits at least two nontrivial weak solutions $\bar{u}_{1}$, $\bar{u}_{2}$, with $\bar{u}_{1}$ such that $\left(\frac{q_{0}}{b-a}\right)^{\frac{p-1}{p}} c_{1}<\left\|\bar{u}_{1}\right\|<\left(\frac{q_{0}}{b-a}\right)^{\frac{p-1}{p}} c_{2}$.

Proof The proof of this theorem is divided into two steps. In the first part, by applying Theorem 2.1, we prove the existence of a local minimum for the functional $\Phi-\lambda \Psi$, where $\Phi(u)$ and $\Psi(u)$ are functionals given in (2.7) for all $u \in X$. Obviously, $\Phi$ and $\Psi$ satisfy all regularity assumptions requested in Theorem 2.1, and the critical points in $X$ of the functional $\Phi-\lambda \Psi$ are exactly the weak solutions of problem (P). To this end, we verify condition (2.4) of Theorem 2.1.

Define the following function $u_{0} \in X$, by setting

$$
u_{0}(x)= \begin{cases}\frac{2 d}{b-a}(x-a) & \text { if } x \in\left[a, \frac{a+b}{2}[\right. \\ d & \text { if } x \in\left[\frac{a+b}{2}, b\right]\end{cases}
$$

and estimate $\Psi\left(u_{0}\right)$ and $\Phi\left(u_{0}\right)$ as follows:

$$
\Psi\left(u_{0}\right) \geq \int_{\frac{a+b}{2}}^{b} F(x, d) d x
$$

and

$$
\Phi\left(u_{0}\right) \leq \frac{1}{p}\left(\frac{2}{b-a}\right)^{p-1}\left[\|q\|_{\infty}+\frac{p+2}{p+1}\left(\frac{b-a}{2}\right)^{p}\|r\|_{\infty}\right] d^{p} .
$$

Fix $c_{1}, d, c_{2}$ satisfying (3.1) and put $r_{1}=\frac{1}{p}\left(\frac{q_{0}}{b-a}\right)^{p-1} c_{1}^{p}$ and $r_{2}=\frac{1}{p}\left(\frac{q_{0}}{b-a}\right)^{p-1} c_{2}^{p}$.

From (3.1), one has $r_{1}<\Phi\left(u_{0}\right)<r_{2}$.

Moreover, for all $u \in X$ such that $u \in \Phi^{-1}(]-\infty, r_{2}[)$, one has

$$
\Psi(u)=\int_{a}^{b} F(x, u(x)) d x \leq \int_{a}^{b} \max _{|\xi| \leq c_{2}} F(x, \xi) d x .
$$


Hence,

$$
\sup _{u \in \Phi^{-1}(]-\infty, r_{2}[)} \Psi(u) \leq \int_{a}^{b} \max _{|\xi| \leq c_{2}} F(x, \xi) d x .
$$

Now, arguing as before, we obtain

$$
\sup _{\left.\left.u \in \Phi^{-1}(]-\infty, r_{1}\right]\right)} \Psi(u) \leq \int_{a}^{b} \max _{|\xi| \leq c_{1}} F(x, \xi) d x .
$$

From hypothesis (i) and bearing in mind (3.3), (3.2), (3.5), and (3.6), we obtain

$$
\beta\left(r_{1}, r_{2}\right)<\rho_{2}\left(r_{1}, r_{2}\right)
$$

From Theorem 2.1, for each $\lambda \in] \frac{1}{a_{d}\left(c_{1}\right)}, \frac{1}{a_{d}\left(c_{2}\right)}\left[, \Phi-\lambda \Psi\right.$ admits at least one critical point $\bar{u}_{1}$ which is a local minimum such that

$$
\left(\frac{q_{0}}{b-a}\right)^{\frac{p-1}{p}} c_{1}<\left\|\bar{u}_{1}\right\|<\left(\frac{q_{0}}{b-a}\right)^{\frac{p-1}{p}} c_{2} .
$$

Now, we prove the existence of the second local minimum distinct from the first one. To this end, we must show that the functional $\Phi-\lambda \Psi$ satisfies the hypotheses of the mountain pass theorem.

Clearly, the functional $\Phi-\lambda \Psi$ is of class $C^{1}$ and $(\Phi-\lambda \Psi)(0)=0$.

From the first part of the proof, we can assume that $\bar{u}_{1}$ is a strict local minimum for $\Phi-\lambda \Psi$ in $X$. Therefore, there is $\rho>0$ such that $\inf _{\left\|u-u_{1}\right\|=\rho}(\Phi-\lambda \Psi)(u)>(\Phi-\lambda \Psi)\left(\bar{u}_{1}\right)$, so condition $\left[13,\left(I_{1}\right)\right.$, Theorem 2.2$]$ is verified.

Now, choosing any $u \in X \backslash\{0\}$, from (ii) one has

$$
\begin{aligned}
(\Phi-\lambda \Psi)(t u) & =\frac{1}{p}\|t u\|^{p}-\lambda \int_{a}^{b} F(x, t u(x)) d x \\
& \leq \frac{t^{p}}{p}\|u\|^{p}-\lambda t^{\mu} a_{3} \int_{a}^{b}|u|^{\mu}+a_{4}(b-a) \rightarrow-\infty
\end{aligned}
$$

as $t \rightarrow+\infty$, so condition [13, $\left(I_{2}\right)$, Theorem 2.2] is verified. Moreover, by standard computations, $\Phi-\lambda \Psi$ satisfies the Palais-Smale condition. Hence, the classical theorem of Ambrosetti and Rabinowitz ensures a critical point $\bar{u}_{2}$ of $\Phi-\lambda \Psi$ such that $(\Phi-\lambda \Psi)\left(\bar{u}_{2}\right)>$ $(\Phi-\lambda \Psi)\left(\bar{u}_{1}\right)$. So, $\bar{u}_{1}$ and $\bar{u}_{2}$ are two distinct weak solutions of $(\mathrm{P})$ and the proof is complete.

Remark 3.1 We observe that in literature the existence of at least one nontrivial solution for differential problems is obtained associating to the classical Ambrosetti-Rabinowitz condition a hypothesis on the nonlinear term of type $f(x, t)=o(|t|)$ as $t \rightarrow 0$. This implies that the problem possesses also the trivial solution $u \equiv 0$. In Theorem 3.1, we find a nontrivial solution of the problem that actually is a proper local minimum of the EulerLagrange functional associated to the problem different from zero.

Now, we present an application of Theorem 2.2 which we will use to obtain multiple solutions. 
Theorem 3.2 Assume that there exist two constants $\bar{c}, \bar{d}$, with $\left(\frac{q_{0}^{p-2}}{2^{p-1}}\right)^{\frac{1}{p}} \bar{c}<\bar{d}$, such that

$$
\int_{a}^{b} \max _{|\xi| \leq \bar{c}} F(x, \xi) d x<\int_{\frac{a+b}{2}}^{b} F(x, \bar{d}) d x
$$

and

$$
\limsup _{|\xi| \rightarrow+\infty} \frac{F(x, \xi)}{|\xi|^{p}} \leq 0 \quad \text { uniformly in } X .
$$

Then, for each $\lambda>\tilde{\lambda}$, where

$$
\tilde{\lambda}=\frac{\bar{r} \bar{d}^{p}-\bar{q} \bar{c}^{p}}{\int_{\frac{a+b}{2}}^{b} F(x, \bar{d}) d x-\int_{a}^{b} \max _{|\xi| \leq \bar{c}} F(x, \xi) d x},
$$

problem $(\mathrm{P})$ admits at least one nontrivial weak solution $\tilde{u}$ such that $\|\tilde{u}\|>\frac{\bar{c}}{\left(\frac{q_{0}}{b-a}\right)^{\frac{p-1}{p}}}$.

Proof The functionals $\Phi$ and $\Psi$ satisfy all regularity assumptions requested in Theorem 2.2. Moreover, by standard computations, condition (3.8) implies that $\Phi-\lambda \Psi, \lambda>0$, is coercive. So, our aim is to verify condition (2.5) of Theorem 2.2. To this end, put

$$
\begin{aligned}
& r=\frac{\bar{c}^{p}}{p}\left(\frac{q_{0}}{b-a}\right)^{p-1}, \text { and } \\
& u_{0}(x)= \begin{cases}\frac{2 \bar{d}}{b-a}(x-a) & \text { if } x \in\left[a, \frac{a+b}{2}[,\right. \\
\bar{d} & \text { if } x \in\left[\frac{a+b}{2}, b\right] .\end{cases}
\end{aligned}
$$

Arguing as in the proof of Theorem 3.1, we obtain that

$$
\rho(r) \geq \frac{\int_{\frac{a+b}{2}}^{b} F(x, \bar{d}) d x-\int_{a}^{b} \max _{|\xi| \leq \bar{c}} F(x, \xi) d x}{\frac{1}{p}\left(\frac{2}{b-a}\right)^{p-1} \overline{d^{p}}\left[\|q\|_{\infty}+\frac{p+2}{p+1}\left(\frac{b-a}{2}\right)^{p}\|r\|_{\infty}\right]-\frac{1}{p}\left(\frac{q 0}{b-a}\right)^{p-1} \bar{c}^{p}} .
$$

So, from our assumption, it follows that $\rho(r)>0$.

Hence, from Theorem 2.2 for each $\lambda>\tilde{\lambda}$, the functional $\Phi-\lambda \Psi$ admits at least one local minimum $\tilde{u}$ such that $\|\tilde{u}\|>\frac{\bar{c}}{\left(\frac{q 0}{b-a}\right) \frac{p-1}{p}}$ and our conclusion is achieved.

Remark 3.2 We point out that the same statement of above given result can be obtained by using a classical direct methods theorem (see [14]), but in addition we get the location of the solution, hence in particular the solution is nontrivial.

Now, we point out some results when the nonlinear term is with separable variables. To be precise, let

- $\alpha \in L^{1}([a, b])$ such that $\alpha(x) \geq 0$ a.e. $x \in[a, b], \alpha \not \equiv 0$, and

- $g: \mathbb{R} \rightarrow \mathbb{R}$ be a nonnegative continuous function,

consider the following boundary value problem:

$$
\left\{\begin{array}{l}
\left.-u^{\prime \prime}=\lambda \alpha(x) g(u) \quad \text { on }\right] a, b[ \\
u(a)=u^{\prime}(b)=0 .
\end{array}\right.
$$


We observe that the following results give the existence of multiple nonnegative solutions since the nonlinear term is supposed to be nonnegative. In order to justify what has been said above, we point out the following weak maximum principle.

Lemma 3.1 Suppose that $\bar{u} \in X$ is a weak solution of problem (P1), then $\bar{u}$ is nonnegative.

Proof We claim that a weak solution $\bar{u}$ is nonnegative. In fact, arguing by a contradiction and setting $A=\{x \in[a, b]: \bar{u}(x)<0\}$, one has $A \neq \emptyset$. Put $\bar{u}^{-}=\min \{\bar{u}, 0\}$, one has $\bar{u}^{-} \in X$ (see, for instance, $[15$, Lemma 7.6]). So, taking into account that $\bar{u}$ is a weak solution and by choosing $v=\bar{u}^{-}$, one has

$$
\int_{A}\left|\bar{u}^{\prime}(x)\right| \bar{u}^{\prime}(x) d x=\lambda \int_{A} \alpha(x) g(\bar{u}(x)) \bar{u}(x) d x \leq 0
$$

that is, $\|\bar{u}\|_{W^{1,2}(A)}=0$ which is absurd. Hence, our claim is proved.

\section{Corollary 3.1 Assume that}

(i') there exist three nonnegative constants $c_{1}, c_{2}, d$, with $c_{1}<\sqrt{2} d<c_{2}$, such that

$$
\frac{\|\alpha\|_{L^{1}([a, b])} G\left(c_{2}\right)-\|\alpha\|_{\left.L^{1}\left(\frac{a+b}{2}, b\right]\right)} G(d)}{c_{2}^{2}-2 d^{2}}<\frac{\|\alpha\|_{L^{1}\left(\left[\frac{a+b}{2}, b\right]\right)} G(d)-\|\alpha\|_{L^{1}([a, b])} G\left(c_{1}\right)}{2 d^{2}-c_{1}^{2}}
$$

(ii') there exist $v>2$ and $R>0$ such that

$$
0<\nu G(t) \leq \operatorname{tg}(t), \quad \text { for all }|t| \geq R \text {. }
$$

Then, for each $\lambda \in \Lambda$, where

$$
\begin{aligned}
\Lambda= & \frac{2 d^{2}-c_{1}^{2}}{2(b-a)\left[\|\alpha\|_{L^{1}\left(\left[\frac{a+b}{2}, b\right]\right)} G(d)-\|\alpha\|_{L^{1}([a, b])} G\left(c_{1}\right)\right]}, \\
& \frac{c_{2}^{2}-2 d^{2}}{2(b-a)\left[\|\alpha\|_{L^{1}([a, b])} G\left(c_{2}\right)-\|\alpha\|_{L^{1}\left(\left[\frac{a+b}{2}, b\right]\right)} G(d)\right]}[
\end{aligned}
$$

problem (P1) admits at least two nonnegative weak solutions $\bar{u}_{1}$ and $\bar{u}_{2}$ such that $\frac{1}{\sqrt{b-a}} c_{1}<$ $\left\|\bar{u}_{1}\right\|<\frac{1}{\sqrt{b-a}} c_{2}$.

Theorem 3.3 Assume that there exist two positive constants $c, d$, with $\sqrt{2} d<c$, such that

$$
\frac{G(c)}{c^{2}}<\left(\frac{\|\alpha\|_{L^{1}\left(\left[\frac{a+b}{2}, b\right]\right)}}{2\|\alpha\|_{L^{1}([a, b])}}\right) \frac{G(d)}{d^{2}} .
$$

Further, suppose that there exist $v>2$ and $R>0$ such that

$$
0<v G(t) \leq \operatorname{tg}(t), \quad \text { for all }|t| \geq R
$$

Then, for each $\lambda \in] \frac{2 d^{2}}{2(b-a)\|\alpha\|_{L^{1}\left(\left[\frac{a+b}{2}, b\right]\right)}^{G(d)}}, \frac{c^{2}}{2(b-a)\|\alpha\|_{L^{1}([a, b])}^{G(c)}}[$, problem (P1) admits at least two nonnegative weak solutions. 
Proof Our aim is to apply Corollary 3.1. To this end, we pick $c_{1}=0$ and $c_{2}=c$. From (3.10), one has

$$
\begin{aligned}
& \frac{\|\alpha\|_{L^{1}([a, b])} G\left(c_{2}\right)-\|\alpha\|_{L^{1}\left(\left[\frac{a+b}{2}, b\right]\right)} G(d)}{c_{2}^{2}-2 d^{2}} \\
& <\frac{\frac{1}{c^{2}}\left[c^{2}\|\alpha\|_{L^{1}([a, b])} G(c)-2 d^{2}\|\alpha\|_{L^{1}([a, b])} G(c)\right]}{c^{2}-2 d^{2}} \\
& \quad=\frac{\|\alpha\|_{L^{1}([a, b])} G(c)}{c^{2}} .
\end{aligned}
$$

On the other hand, one has

$$
\frac{\|\alpha\|_{L^{1}\left(\left[\frac{a+b}{2}, b\right]\right)} G(d)-\|\alpha\|_{L^{1}([a, b])} G\left(c_{1}\right)}{2 d^{2}-c_{1}^{2}}=\frac{\|\alpha\|_{L^{1}\left(\left[\frac{a+b}{2}, b\right]\right)} G(d)}{2 d^{2}} .
$$

Hence, from Corollary 3.1 and taking (2.6) into account, the conclusion follows.

A further consequence of Theorem 3.1 is the following result.

\section{Theorem 3.4 Assume that}

$$
\lim _{t \rightarrow 0^{+}} \frac{g(t)}{t}=+\infty
$$

and there are constants $\mu>2$ and $R>0$ such that, for all $|\xi| \geq R$, one has

$$
0<\mu G(\xi) \leq \xi g(\xi) .
$$

Then, for each $\lambda \in] 0, \lambda^{*}\left[\right.$, where $\lambda^{*}=\frac{1}{2(b-a)\|\alpha\|_{L^{1}([a, b])}} \sup _{c>0} \frac{c^{2}}{G(c)}$, problem (P1) admits at least two nonnegative weak solutions.

Proof Fix $\lambda \in] 0, \lambda^{*}\left[\right.$. Then there is $c>0$ such that $\lambda<\frac{1}{2(b-a)\|\alpha\|_{L^{1}([a, b])}} \frac{c^{2}}{G(c)}$. From (3.11), there is $d<\frac{c}{\sqrt{2}}$ such that $\frac{2(b-a)\|\alpha\|_{L^{1}\left(\left[\frac{a+b}{2}, b\right]\right)} G(d)}{2 d^{2}}>\frac{1}{\lambda}$. Hence, Theorem 3.3 ensures the conclusion.

Next, as a consequence of Theorems 3.3 and 3.2, the following theorem of the existence of three solutions is obtained.

Theorem 3.5 Assume that

$$
\limsup _{|\xi| \rightarrow+\infty} \frac{G(\xi)}{\xi^{2}} \leq 0 .
$$

Moreover, assume that there exist four positive constants $c, d, \bar{c}, \bar{d}$, with $\sqrt{2} d<c \leq \bar{c}<\sqrt{2} \bar{d}$, such that (3.10),

$$
G(\bar{c})<\left(\frac{\|\alpha\|_{L^{1}\left(\left[\frac{a+b}{2}, b\right]\right)}}{\|\alpha\|_{L^{1}([a, b])}}\right) \frac{1}{2} G(\bar{d}),
$$


and

$$
\frac{G(c)}{c^{2}}<\frac{\|\alpha\|_{L^{1}\left(\left[\frac{a+b}{2}, b\right]\right)} G(\bar{d})-\|\alpha\|_{L^{1}([a, b])} G(\bar{c})}{\frac{1}{b-a} \bar{d}^{2}-\frac{1}{2(b-a)} \bar{c}^{2}}
$$

are satisfied.

Then, for each $\lambda \in \Lambda=] \max \left\{\tilde{\lambda}, \frac{2 d^{2}}{2(b-a)\|\alpha\|_{L^{1}\left(\left[\frac{a+b}{2}, b\right]\right)}^{G(d)}}\right\}, \frac{c^{2}}{2(b-a)\|\alpha\|_{L^{1}([a, b])} G(c)}[$, problem (P1) admits at least three weak nonnegative solutions.

Proof First, we observe that $\Lambda \neq \emptyset$ owing to (3.13). Next, fix $\lambda \in \Lambda$. Theorem 3.3 ensures a nontrivial weak solution $\bar{u}$ such that $\|\bar{u}\|<c$ which is a local minimum for the associated functional $\Phi-\lambda \Psi$, as well as Theorem 3.2 guarantees a nontrivial weak solution $\tilde{u}$ such that $\|\tilde{u}\|>c$ which is a local minimum for $\Phi-\lambda \Psi$. Hence, the mountain pass theorem as given by Pucci and Serrin (see [2]) ensures the conclusion.

\section{Theorem 3.6 Assume that}

$$
\begin{aligned}
& \limsup _{\xi \rightarrow 0^{+}} \frac{G(\xi)}{\xi^{2}}=+\infty, \\
& \limsup _{\xi \rightarrow+\infty} \frac{G(\xi)}{\xi^{2}}=0 .
\end{aligned}
$$

Further, assume that there exist two positive constants $\bar{c}, \bar{d}$, with $\bar{c}<\sqrt{2} \bar{d}$, such that

$$
\frac{G(\bar{c})}{\bar{c}^{2}}<\left(\frac{\|\alpha\|_{L^{1}\left(\left[\frac{a+b}{2}, b\right]\right)}}{2\|\alpha\|_{L^{1}([a, b])}}\right) \frac{G(\bar{d})}{\bar{d}^{2}} .
$$

Then, for each $\lambda \in] \frac{2 \bar{d}^{2}}{2(b-a)\|\alpha\|_{L^{1}\left(\left[\frac{a+b}{2}, b\right]\right)} G(\bar{d})}, \frac{\bar{c}^{2}}{2(b-a)\|\alpha\|_{L^{1}([a, b])} G(\bar{c})}[$, problem (P1) admits at least three weak nonnegative solutions.

Proof Clearly, (3.15) implies (3.8). Moreover, by choosing $d$ small enough and $c=\bar{c}$, simple computations show that (3.14) implies (3.10). Finally, from (3.16) we get (3.7) and also (3.13). Hence, Theorem 3.5 ensures the conclusion.

Finally, we present two examples of problems that admit multiple solutions owing to Theorems 3.4 and 3.6.

Example 3.1 Owing to Theorem 3.4, for each $\lambda \in] 0, \frac{1}{2}[$, the problem

$$
\left\{\begin{array}{l}
\left.-u^{\prime \prime}=\lambda\left(u^{4}+1\right) \quad \text { in }\right] 0,1[ \\
u(0)=u^{\prime}(1)=0
\end{array}\right.
$$

admits at least two nonnegative solutions. In fact, one has $\lim _{u \rightarrow 0^{+}} \frac{g(u)}{u}=\lim _{u \rightarrow 0^{+}} \frac{u^{4}+1}{u}=$ $+\infty$ and $(A R)$ is satisfied as a simple computation shows. Moreover, one has $\lambda^{*}=$ $\frac{1}{2(b-a)\|\alpha\|_{1}} \sup _{c>0} \frac{c^{2}}{G(c)}=\frac{1}{2}$. 
Example 3.2 Consider the following problem:

$$
\left\{\begin{array}{l}
\left.-u^{\prime \prime}=\frac{1}{10}\left(\frac{u^{7}}{e^{u}}+1\right) \quad \text { in }\right] 0,1[ \\
u(0)=u^{\prime}(1)=0 .
\end{array}\right.
$$

It has three nonnegative solutions. In fact, let $g: \mathbb{R} \rightarrow \mathbb{R}$ be a function defined as

$$
g(u)= \begin{cases}\left(\frac{u^{7}}{e^{u}}+1\right) & \text { if } u \geq 0 \\ 1 & \text { if } u<0 .\end{cases}
$$

Owing to Theorem 3.6, the following problem

$$
\left\{\begin{array}{l}
\left.-u^{\prime \prime}=\frac{1}{10} g(u) \quad \text { in }\right] 0,1[, \\
u(0)=u^{\prime}(1)=0
\end{array}\right.
$$

admits three nonnegative classical solutions. In fact, one has

$$
\lim _{u \rightarrow 0^{+}} \frac{g(u)}{u}=\lim _{u \rightarrow 0^{+}} \frac{\left(\frac{u^{7}}{e^{u}}+1\right)}{u}=+\infty \text { and } \lim _{u \rightarrow+\infty} \frac{g(u)}{u}=\lim _{u \rightarrow+\infty} \frac{\left(\frac{u^{7}}{e^{u}}+1\right)}{u}=0 .
$$

Moreover, taking into account that $G(u)=u-\frac{\sum_{i=0}^{7} \frac{7 !}{i} u^{i}}{e^{u}}+7$ !, by choosing $\bar{c}=2$ and $\bar{d}=9$, one has $\frac{G(2)}{2^{2}}<\frac{1}{4} \frac{G(9)}{9^{2}}$ and $\frac{29^{2}}{G(9)}<\frac{1}{10}<\frac{2^{2}}{2 G(2)}$.

So, it is clear that any nonnegative solution $u$ of problem $\left(P_{g}\right)$ is also a solution of problem $\left(P_{E}\right)$.

\section{Competing interests}

The author declares that she has no competing interests.

Received: 20 July 2012 Accepted: 26 October 2012 Published: 14 November 2012

\section{References}

1. D'Aguì, G: Existence results for a mixed boundary value problem with Sturm-Liouville equation. Adv. Pure Appl. Math. 2, 237-248 (2011)

2. Pucci, P, Serrin, J: A mountain pass theorem. J. Differ. Equ. 63, 142-149 (1985)

3. Agarwal, RV, O'Regan, D, Wong, PJY: Positive Solutions of Differential, Difference and Integral Equations. Kluwer Academic, Dordrecht (1999)

4. Bonanno, G, Sciammetta, A: Existence and multiplicity results to Neumann problems for elliptic equations involving the $p$-Laplacian. J. Math. Anal. Appl. 390, 59-67 (2012)

5. Averna, D, Giovannelli, N, Tornatore, E: Existence of three solutions for a mixed boundary value problem with the Sturm-Liouville equation. Preprint

6. Averna, D, Salvati, R: Three solutions for a mixed boundary value problem involving the one-dimensional $p$-Laplacian. J. Math. Anal. Appl. 298, 245-260 (2004)

7. Lü, H, O'Regan, D, Zhong, C: Multiple positive solutions for the one-dimensional singular p-Laplacian. Appl. Math Comput. 133, 407-422 (2002)

8. Salvati, R: Multiple solutions for a mixed boundary value problem. Math. Sci. Res. J. 7, 275-283 (2003)

9. Bonanno, G: A critical point theorem via the Ekeland variational principle. Nonlinear Anal. 75, 2992-3007 (2012)

10. Ricceri, B: A general variational principle and some of its applications. J. Comput. Appl. Math. 113, 401-410 (2000)

11. Bonanno, G, Candito, P: Non-differentiable functionals and applications to elliptic problems with discontinuous nonlinearities. J. Differ. Equ. 244, 3031-3059 (2008)

12. Bonanno, G, Marano, SA: On the structure of the critical set of non-differentiable functions with a weak compactness condition. Appl. Anal. 89, 1-10 (2010)

13. Rabinowitz, PH: Minimax Methods in Critical Point Theory with Applications to Differential Equations. CBMS Reg. Conf. Ser. Math., vol. 65. Am. Math. Soc., Providence (1986)

14. Struwe, M: Variational Methods. Springer, Berlin (1996)

15. Gilbarg, D, Trudinger, NS: Elliptic Partial Differential Equations of Second Order, 2nd edn. Springer, Berlin (1983) 
doi:10.1186/1687-2770-2012-134

Cite this article as: $D$ 'Agui: Multiplicity results for nonlinear mixed boundary value problem. Boundary Value Problems 2012 2012:134.

Submit your manuscript to a SpringerOpen ${ }^{\circ}$ journal and benefit from:

- Convenient online submission

- Rigorous peer review

- Immediate publication on acceptance

Open access: articles freely available online

- High visibility within the field

- Retaining the copyright to your article

Submit your next manuscript at $\boldsymbol{s p r i n g e r o p e n . c o m ~}$ 